\title{
Update on cervical disc arthroplasty: where are we and where are we going?
}

\author{
Jorge J. Jaramillo-de la Torre · Jonathan N. Grauer • \\ James J. Yue
}

Published online: 26 February 2008

(C) Humana Press 2008

\begin{abstract}
Despite the very good results of anterior cervical discectomy and fusion, there are concerns of adjacent level degeneration. For this reason, interest has grown in the potential for motion sparing alternatives. Cervical disc arthroplasty is thus evolving as a potential alternative to fusion. Specific design characteristic and implants will be reviewed and outcomes summarized.
\end{abstract}

Keywords Cervical vertebrae - Spine - Arthroplasty · Fusion

\section{Introduction}

Anterior cervical discectomy and fusion (ACDF) for degenerative disc disease has been widely performed since the initial description of the procedure in the late 1950s [1]. The procedure has been used to address degenerative changes and neural element compression with favorable rates of fusion and successful clinical outcomes in $85-95 \%$ of the patients [2, 3] (Fig. 1).

In the cervical spine with disc herniation or spondylosis causing radiculopathy and/or myelopathy, the decision of performing an anterior decompression is independent of the type of reconstruction that will follow. After the decompression is accomplished, a reconstruction is performed to avoid postoperative pain and kyphosis. This reconstruction has typically been with a fusion but total

J. J. Jaramillo-de la Torre · J. N. Grauer $(\bowtie) \cdot$ J. J. Yue Division of Spine Surgery, Department of Orthopaedics and Rehabilitation, Yale University School of Medicine, P.O. Box 208071, New Haven, CT 06520-8071, USA

e-mail: jonathan.grauer@yale.edu disc replacement (TDR) is evolving as a potential alternative [4] (Figs. 2 and 3).

\section{Rationale of cervical arthroplasty}

Adjacent segment degeneration

Fusion of a mobile spinal segment may lead to adverse effects such as accelerated degeneration of the adjacent motion segment [5]. Biomechanical studies have shown that cervical fusion alters the adjacent level kinematics [6]. It has been demonstrated that after an ACDF, the loss of motion at the fused level is compensated by an increase in motion [7] as well as an increase in intradiscal pressure at adjacent levels [8].

Adjacent segment degeneration (ASD) is defined as radiographic changes at a segment adjacent to a fusion and is not necessarily associated with symptoms. Adjacent level disease, as opposed to simple degeneration, may be associated with pain, radicular, or myelopathic signs and/or symptoms [2].

Hilibrand et al. [9] reported the incidence of symptomatic ASD to be $2.9 \%$ per year after ACDF in an often quoted study. Survivorship analysis projected that a $25.6 \%$ of the patients who underwent an ACDF would develop symptomatic ASD within 10 years after. The incidence of ASD was higher at the C5-6 and C6-7 levels. However, of note, this study did find that longer constructs did have a lesser incidence of adjacent level degenerative changes and raised the question of weather the affect was due to increased loads of the adjacent level or the progression of the natural history of degenerative disc disease. Goffin et al. [10] also showed a $92 \%$ incidence of radiographic ASD 5-year follow-up after ACDF. The re-operation rate 


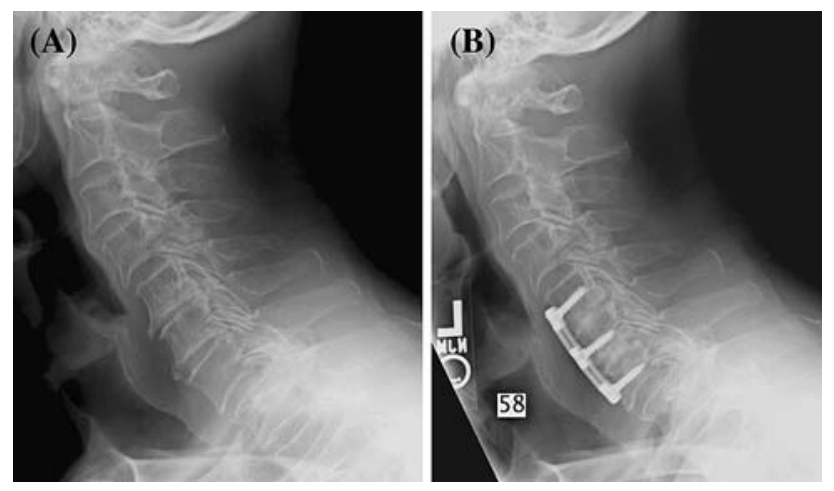

Fig. 1 Lateral cervical radiograph of a patient with multilevel spondylosis (a) for which an ACDF was performed at C56 and C67 for radicular symptoms (b)

because of symptomatic ASD not responding to a longtime conservative therapy was $6.11 \%$. Overall, cervical studies do suggest that there is load concentration and degeneration adjacent to a fusion.

Alternatively, it has been argued that the relatively high frequency of ASD and additional surgery may actually reflect the natural history of cervical spondylosis. We do know that after age 40 almost $60 \%$ of the population will have radiographic evidence of degenerative changes, and after age $6595 \%$ of men and $70 \%$ of women have radiographic evidence of degenerative changes [4]. To that point, patients followed after posterior laminoforaminotomy (without fusion) have been shown to have an incidence of ASD of $3.9 \%$ per year [11]. This is in the range of that described for ACDF in a population without fusion and thus for whom there would not be expected increased loading at the adjacent levels.

In response to the question of ASD as a result of the natural history of cervical spondylosis, Goffin et al. [12] studied the development of radiological ASD in patients who underwent fusion with anterior plating for trauma. Over a mean follow-up of 7 years, $60 \%$ of patients who had undergone fusion had radiographic evidence of ASD. None of these changes manifested clinically with radiculopathy or myelopathy and thus were not treated surgically. In another study, Goffin et al. [10] showed a 92\% incidence of radiologic ASD at 5-year follow-up after ACDF for cervical spondylosis and trauma patients. The increase in degeneration was statistically equally distributed among younger trauma cases and older non-trauma cases operated mainly for disc herniation or spondylosis. This argues back to the point that symptomatic ASD may be accelerated by fusion procedures.

\section{Potential role of cervical disc arthroplasty}

Although there is some conflicting evidence regarding the incidence of symptomatic ASD, cervical disc arthroplasty has been advocated as a potential alternative to fusion to avoid this potential complication [13]. Normal motion between two vertebrae occurs around a point described as instantaneous axis of rotation (IAR). The location of the
Fig. 2 Lateral cervical radiograph (a) of a patient with a disc herniation at the $\mathrm{C} 67$ level. Prodisc-C was performed and lateral (b), flexion (c), and extension films (d) are shown
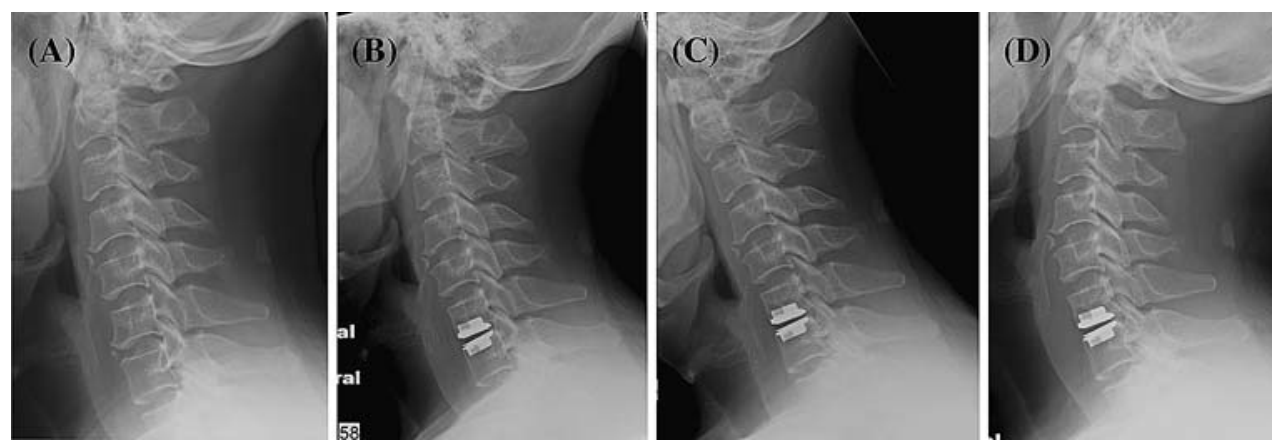

Fig. 3 Lateral cervical radiograph with degenerative changes at CC56 and C67 (a). Two-level discovery arthroplasty was performed and postoperative lateral (b), flexion (c), and extension (d) films are shown
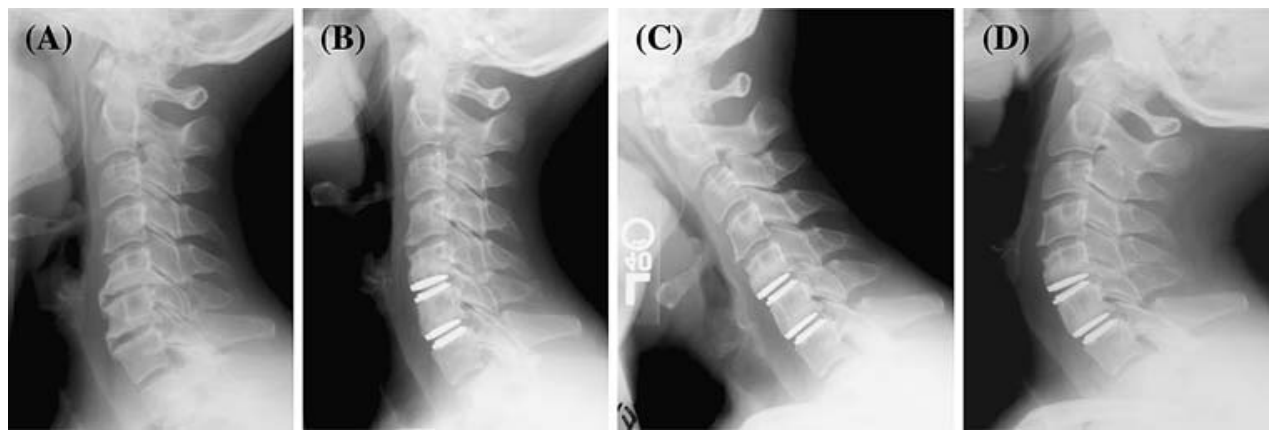
IAR varies between levels but it is generally located in the posterior half of the upper portion of the inferior vertebral body. In order to protect the facet joints from abnormal stresses, cervical arthroplasty devices should have an axis of rotation that mimics the kinematics of the normal spine. They should also restore physiologic range of motion, be able to restore disc height, and transmit axial loading forces from the superior vertebral body to the one inferior [14].

Biomechanical studies have suggested that TDR may allow for a more normal restoration of load transfer and kinematics at adjacent levels when compared with fusion [7]. After TDR, the stress profiles in specimens at the adjacent level discs were similar to those of intact, nontreated levels, with reduced stresses in the adjacent level annulus when compared with spines with simulated fusions [15].

Robertson et al. [16] conducted a study to compare the incidence of radiological documented changes and symptomatic adjacent-level cervical disc disease after single-level discectomy and subsequent cervical fusion or arthroplasty using the Bryan disc. This study showed the appearance of new radiographic changes in $34.6 \%$ of the fusion-treated patients and in $17.5 \%$ of the arthroplastytreated patients at 24 months $(P=0.009)$. New symptomatic adjacent degenerative disc disease occurred in $7 \%$ of the fusion group and in none of the arthroplasty group $(P=0.018)$. This study showed that maintaining motion with arthroplasty after single-level anterior discectomy rather than fusion will delay or prevent symptomatic postoperative disc disease and will decrease to a significant degree the associated radiological disc degeneration [16].

\section{Treatment of symptomatic ASD}

The management of symptomatic ASD that requires surgical intervention is more challenging than primary pathology. The surgical risks such as dysphagia and pseudoarthrosis are increased as compared to index surgical procedures [17]. To some extent, this is due to the need to re-approach the anterior cervical region (something that may be done from the same or opposite side). Additionally, the biomechanics of being adjacent to an already fused level increases the mechanical loads at an adjacent level site. Moreover, the success rate of ACDF decreases with an increase in the number of levels fused [17].

Because the success of cervical TDR does not rely on a biological fusion process, it may be a more effective treatment strategy for ASD [18]. In fact, there is an evidence of good results in patients with symptomatic ASD after ACDF or congenital fusion treated with arthroplasty [19]. Furthermore, TDR has the patient appeal of not beginning a gradual adding on fusion process.
Decrease of morbidity associated with ACDF and potentially hastened recovery

There are certainly potential morbidities associated with any surgical intervention. ACDF has long been associated with the morbidity of bone graft harvest, including pain, infection, meralgia paresthetica, and pelvic fracture [18, 20]. This has largely decreased as other bone graft materials such as allograft, cages, etc., have become more standard, especially for short constructs.

There is additionally the potential of pseudoarthrosis with fusion procedures. This may not be symptomatic even if it occurs, but motion and potential collapse may be associated with axial pain or neural symptoms which may require further attention.

Further, many surgeons place patients in external immobilization collars after ACDF. This is not well supported by the literature in an era where most are using anterior cervical instrumentation, but this is cited as a significant issue by many patients. With TDR, the duration of postoperative immobilization is decreased, which facilitates a faster return to daily activities [20]. To this end, Mummaneni et al. [21] showed that patients who underwent cervical TDR returned to work 16 days sooner than those who underwent ACDF.

\section{Indications and contraindications for cervical TDA}

The quoted indications for cervical TDA are largely drawn from the clinical trials that have been used to assess their safety and efficacy. Within trials, there must be "on label" uses for the devices only. However, as cervical arthroplasty devices become available as approved devices, the indications for which they are considered continue to expand.

Most disc arthroplasty devices have "on label" indications for patients with degenerative disc disease who have failed conservative treatment and require surgical intervention at one or two levels from $\mathrm{C} 3$ to $\mathrm{T} 1$ for symptoms and signs of radiculopathy or myelopathy with or without axial neck pain secondary to disc herniation or spondylosis $[18,22]$.

Sekhon [23] reported a series of 11 patients and 15 artificial disc prostheses studying Bryan cervical disc for the treatment of cervical spondylotic myelopathy. He reported that $91 \%$ of patients had a good or excellent outcome using Odom's criteria and a statistically significant decrease in Nurick myelopathy scores at a mean follow-up of 18 months.

Wang et al. [24] reported a series of patients with cervical spondylotic myelopathy using the Bryan disc prosthesis. All of the 65 patients (77 levels) with at least 12 months of follow-up showed improvement according to 
the Odom's criteria (47/65 excellent, $18 / 65$ good). The average JOA score of the 65 patients increased from 8.7 to 15.5 at last follow-up.

Alternative indications for arthroplasty may be considered. For example, three or more levels with degenerative disc disease and the treatment of symptomatic ASD after a fusion may be appropriately considered for TDA [18]. A study reported by Pimenta et al. [25] investigating multilevel cervical arthroplasty versus single-level cervical arthroplasty using the porous-coated motion (PCM) prosthesis showed significantly improved clinical outcomes for multilevel TDR compared to single-level TDR.

Conditions generally agreed to be contraindications for this procedure include: instability on dynamic radiographs, facet arthropathy, osteoporosis, infection, prior laminectomy, primarily axial neck pain, rheumatoid arthritis, ankylosing spondylitis, ossification of the posterior longitudinal ligament (PLL), and diffuse idiopathic skeletal hyperostosis [3, 18, 22]. Multi-level cervical disc arthroplasty has not been evaluated prospectively and appropriate bone density values should be ensured pre-operatively. Careful CT scan assessment of facet joints should be performed to exclude patients with facet degeneration of clinical significance.

\section{Surgical considerations for cervical TDA}

Many surgeons are familiar with ACDF procedures. However, there are specific surgical considerations which must be given to cervical TDA in making the transition from one procedure to the other.

For example, a complete discectomy is needed with complete removal of all osteophytes. As there will continue to be motion, one must be certain to avoid the potential of dynamic compression in the foramen which is not a consideration with ACDF. Further, there has long been the concept that residual osteophytes will resorb after a fusion and this will not be the case after TDA.

Most believe that the PLL should be removed with TDA even though this is not always done with ACDF. Not only does this ensure that a complete decompression has been achieved, but also the resection of the PLL ensures that the disc space has been mobilized and facilitates parallel distraction, restoration of the intervertebral height, and mobility of the segment.

Although the cartilaginous endplate is removed for TDA, the bony endplate is preserved as possible to minimize the risk of implant subsidence. The vertebral endplates should be burred until there are two parallel surfaces to facilitate even the insertion of the device and to allow appropriate surface contact between the endplates and the device.
With implantation of a cervical TDA, proper midline identification and placement of the device is critical. This is in sharp distinction to a graft for ACDF which can be placed eccentrically or a cervical TDA will not function as designed and allow restoration of motion. We therefore advocate careful fluoroscopic evaluation to ensure that proper visualization of the index disc space can be achieved. If due to body habitus it is not possible to clearly visualize the interspace, strong consideration for fusion should be made.

Finally, after the implantation of the device, over-distraction of the interbody space should be avoided since it may lead to nerve root stretch, facet joint overload, and/or loss of motion [26-28].

\section{Design considerations}

The Cervical Spine Study Group developed a new nomenclature system for cervical arthroplasty. Currently, cervical arthroplasty devices can be classified as nonarticulating, uniarticulating, or biarticulating. The devices are either modular (meaning that they have replaceable components) or they are non-modular (meaning that they have non-replaceable components) [29].

Devices are considered constrained in certain planes if they restrict motion to less than that seen physiologically. Devices are considered semi-constrained in certain planes if they allow motion similar to that seen physiologically. Devices are considered non-constrained in certain planes of motion if there is no mechanical stop to the motion and are reliant on the perispinal soft tissue, and the inherent compression across the disc space to provide restraint to extremes of motion [5]. Some devices have inherent coupling of motion in different planes, whereas others do not.

Prostheses are made of several components, each of which has specific functional considerations which are affected by material, design, articulation, etc. Components must remain permanently affixed to the vertebral endplate and wear characteristics must be optimized [4].

Implant endplates must be made of durable, non-reactive metals that can be molded to the desired form. Cobaltchrome is commonly considered as biocompatible with advantageous mechanical properties. However, this does significantly interfere with potential future imaging studies such as CT and MRI $[14,30]$. Stainless steel is similarly biocompatible and less expensive to fabricate but has a high modulus of elasticity which may be related to subsidence. Titanium is the other biocompatible material which is often considered as it has a modulus of elasticity most similar to bone and advantageous to future MRI imaging $[4,31]$; however, this is more susceptible to notching and wear. Surface treatment of titanium, such as coating with 
nitride or diamond-like carbon, may improve hardness and wear characteristics [4].

The distribution of force and subsidence is possibly the most important biomechanical considerations for an artificial disc. The idea is to distribute the forces involved as uniformly as possible over a large area [30]. The interface between prosthesis and vertebra should allow transmission of axial forces between adjacent vertebrae [14].

The initial stability of the prosthesis may be accomplished by some form of anchoring of the implant to the vertebral body by screws, fins, or keels. Long-term stability typically implies osteointegration of the device into surrounding bone. Implant surface coatings to encourage bone ingrowth include titanium wire mesh, plasma-sprayed titanium, porous cobalt-chrome, and bioactive materials such as hydroxyapatite and calcium phosphate. Initial and long-term fixations should prevent subluxation, subsidence, or displacement [4, 14].

The articular surfaces are the other very important design consideration for any arthroplasty device. Most commonly this has been metal on polymer such ultra-high molecular weight polyethylene (UHMWPE) or polyurethane [14]. This articulation provides a low-friction articulation. There has been significant concern of resultant polyethylene debris, but this has not been found to be a significant clinical problem. Alternatively, metal-on-metal or ceramic articulation may be considered. These have potentially lower wear rates compared with polyethylene-on-metal articulations but provide less shock absorption [4].

\section{Specific implants}

\section{Metal-on-metal devices}

The Prestige ST cervical disc (Medtronic Sofamor Danek) is a stainless steel on stainless steel device consists of two articulating components in a ball-and-trough articulation. It was approved by the FDA on July 2007. It is attached to the cervical vertebrae with screws. The design of the device provides relatively unconstrained motion. The surfaces of the device contacting the endplates are grit-blasted to promote bone osteointegration [26]. The Prestige LP is the most recent version of the Prestige family of cervical discs. It radically differs from its predecessors in that acute fixation is achieved by a set of rails that are placed on the intervertebral contact surface. It is manufactured from a titanium ceramic composite material, and a porous titanium plasma spray coating on the endplate surface facilitates bone in-growth and long-term fixation [26, 29, 32].

The CerviCore cervical disc (Striker Spine) is constructed of chrome-cobalt design with a saddle-shaped articulation. The device is attached to vertebral bodies by screws. The base plates feature a titanium spray and three spikes. The designers assert that the device mimics the normal disc axis of rotation and the function of the uncovertebral articulation and promotes vertebral foraminal widening during coupled rotation and bending. There are no reports of clinical implantation of this device [4].

\section{Metal-on-polymer devices}

The ProDisc-C cervical disc (Synthes Spine Solutions) was approved by the FDA on December 2007. It consists of cobalt-chromium alloy endplates with a central keel for anchorage to the vertebral bodies and a locking core of UHMWPE as a central polymer that provides a ball-andsocket articulation. The endplates are coated with a titanium plasmapore for tissue compatibility and bone ingrowth. It is considered constrained in compression, unconstrained in distraction and rotation, and semi-constrained in flexion, extension, and lateral bending [27, 33].

The Bryan cervical disc (Medtronic Sofamor Danek) was designated to preserve normal kinematics with an axially symmetric prosthesis that mimics normal flexion, extension, and lateral bending. It consists of a single-piece of a porous-coated, clamshell-shaped titanium endplates with a polycarbonate polyurethane core. A polyurethane membrane that surrounds the articulation reduces friction and contains debris [20]. The device provides a relatively unconstrained range of motion [28].

The PCM prosthesis (Cervitech) has a large-radius UHMWPE core with cobalt-chrome endplates which are coated with titanium with electrochemically coated calcium phosphate that encourages bone integration. The lowprofile PCM prosthesis is used when the PLL is preserved. In cases when the PLL have been removed as part of the decompression, the fixed PCM implant is preferred. The fixed implant incorporates anterior flanges and screws for a better stability [4, 20].

Secure ${ }^{\circledR}-\mathrm{C}$ Cervical Artificial Disc (Globus Medical) consists of a central UHMWPE component and two titanium plasma-sprayed cobalt-chromium-molybdenum alloy endplates. It is considered a semi-constrained device. The porous-coated bone-contacting surfaces promote osseous ingrowth. The moving axis of rotation allows for shear translation. The device is under the IDE granted by the FDA.

Other devices include CerPass (NuVasive), a ceramicon-ceramic prosthesis; NeoDisc (NuVasive), comprised of a solid silicone core surrounded by a digitally embroidered polyester jacket that is attached to the vertebral bodies with titanium alloy screws; Mobi-C (LDR Médical), comprised of two metal spinal plates and an UHMWPE mobile insert; Kineflex-C (Spinal Motion), a metal-on-metal prosthesis; Discover (DePuy Spine), a metal-on-polymer device; Discocerv (Scient'x), comprised of titanium alloy endplates 
embedded with ceramic bearing components; Catalina (SeaSpine), a ceramic-on-ceramic prothesis; Rescue (Biomet/EBI), a pyrocarbon-on-pyrocarbon prosthesis; Physio$\mathrm{C}$ (Nexgen Spine), a non-articulating metal-polymer prosthesis; Cervidisc (Scient'x), a ceramic-on-ceramic prosthesis; SaluDisc (SpineMedica), a non-articulating hydrogel prosthesis; CMP (Vertebron), a metal-on-polyethylene-on metal prosthesis [34].

\section{Clinical outcomes}

One- and two-year clinical outcome data indicate that cervical TDR is at least as effective as standard ACDF [3542]. Mummaneni et al. [21] reported the results of a prospective randomized multi-center study in which the results of cervical disc arthroplasty using the Prestige ST were compared with ACDF in patients treated for symptomatic single-level cervical degenerative disc disease. They found that the device maintained physiological segmental motion at 24 months after implantation. At last follow-up, all treated levels were mobile with a mean flexion-extension difference of $7.59^{\circ}$. The investigational group showed improved neurological success, improved clinical outcomes, and a reduced rate of secondary surgeries compared with ACDF.

Sasso et al. [43] reported the results of a prospective, randomized, controlled, multi-center trial with 24-month follow-up comparing the outcomes of cervical arthroplasty using the Bryan cervical disc prosthesis to ACDF for patients with single-level symptomatic cervical radiculopathy or myelopathy refractory to non-operative interventions. They found that the investigational group had statistically significant improvements as assessed by the Neck Disability Index, the Neck Pain Score, and the SF-36 Physical component scores. Arm pain relief was similar in both the investigational and the control groups. The disc replacement group retained an average of $7.0^{\circ}$ of angular motion at the target level at 24 months.

Other studies have reported a significant improvement in pain and functional outcome in patients treated with distinct TDR prostheses at 12-18 months [44-46] and 4 years of follow-up with preservation of motion and without development of ASD [47].

Extended follow-up studies with larger patient populations are required to evaluate the efficacy, safety, and ability to prevent the ASD of cervical TDR.

\section{Complications}

Few early complications have been reported after cervical TDR [14]. Pickett et al. [48] reported on the complications in patients treated with Bryan prosthesis. They implanted 96 discs in 74 patients and found a perioperative complication rate of $6.2 \%$ per treated level. The rate of late-onset complications was $5.2 \%$ per treated level and includes two cases of heterotopic ossification, one prosthesis migration, one reoperation for severe segmental kyphosis, and one failure of the prosthesis in extension. Segmental motion was preserved in $96 \%$ or the cases.

Accelerated wear and particle debris formation leading to loosening of the device does not appear to be an issue [14]. Wear testing indicates that these devices will have a long life once implanted [49]. Long-term evaluation is needed to evaluate this statement.

No cases of subsidence of cervical arthroplasty devices have been reported to date [18].

\section{Future directions}

It is expected that motion preservation after cervical TDR will decrease the incidence of ASD. Longer-term follow-up studies are needed to assess this issue. Other points to be considered are the percentage of spontaneous fusion over time, the limitations in restoring abnormal curves, and the cost of the prostheses.

As experience is increased, additional potential indications may emerge such as neck pain, deformity correction, or revision of previous fusion [3].

The continuing research in cervical spine biomechanics, biomaterial science, and surgical technique will probably offer in the future alternative prosthesis with better designs for cervical arthroplasty.

\section{References}

1. Robinson R, Walker A, Ferlic D. The results of anterior interbody fusion of the cervical spine. J Bone Joint Surg Am 1962;44:156987.

2. Bartolomei JC, Theodore N, Sonntag VK. Adjacent level degeneration after anterior cervical fusion: a clinical review. Neurosurg Clin N Am. 2005;16(4):575-87.

3. Mehren C, Mayer HM. Artificial cervical disc replacement-an update. Neurol India 2005;53(4):440-4.

4. Phillips FM, Garfin SR. Cervical disc replacement. Spine 2005;30(17 Suppl):S27-33.

5. Puttlitz CM, DiAngelo DJ. Cervical spine arthroplasty biomechanics. Neurosurg Clin N Am 2005;16(4):589-94.

6. Maiman DJ, Kumaresan S, Yoganandan N, et al. Biomechanical effect of anterior cervical spine fusion on adjacent segments. Biomed Mater Eng 1999;9(1):27-38.

7. DiAngelo DJ, Roberston JT, Metcalf NH. Biomechanical testing of an artificial cervical joint and an anterior cervical plate. $\mathbf{J}$ Spinal Disord Tech 2003;16(4):314-23.

8. Eck JC, Humphreys SC, Lim TH, et al. Biomechanical study on the effect of cervical spine fusion on adjacent-level intradiscal pressure and segmental motion. Spine 2002;27(22):2431-4. 
9. Hilibrand AS, Carlson GD, Palumbo MA, et al. Radiculopathy and myelopathy at segments adjacent to the site of a previous anterior cervical arthrodesis. J Bone Joint Surg Am 1999;81(4):519-28.

10. Goffin J, Geusens E, Vantomme N, et al. Long-term follow-up after interbody fusion of the cervical spine. J Spinal Disord Tech 2004;17(2):79-85.

11. Henderson CM, Hennessy RG, Shuey HM, et al. Posterior-lateral foraminotomy as an exclusive operative technique for cervical radiculopathy: a review of 846 consecutively operated cases. Neurosurgery 1983;13(5):504-12.

12. Goffin J, van Loon J, Van Calenbergh F, et al. Long-term results after anterior cervical fusion and osteosynthetic stabilization for fractures and/or dislocations of the cervical spine. J Spinal Disord 1995;8(6):500-8.

13. Albert TJ, Eichenbaum MD. Goals of cervical disc replacement. Spine J 2004;4(6 Suppl):292S-3S.

14. Sekhon LH, Ball JR. Artificial cervical disc replacement: principles, types and techniques. Neurol India 2005;53(4):445-50.

15. Wigfield CC, Skrzypiec D, Jackowski A, et al. Internal stress distribution in cervical intervertebral discs: the influence of an artificial cervical joint and simulated anterior interbody fusion. $\mathbf{J}$ Spinal Disord Tech 2003;16(5):441-9.

16. Robertson JT, Papadopoulos SM, Traynelis VC. Assessment of adjacent-segment disease in patients treated with cervical fusion or arthroplasty: a prospective 2-year study. J Neurosurg Spine 2005;3(6):417-23.

17. Hilibrand AS, Yoo JU, Carlson GD, et al. The success of anterior cervical arthrodesis adjacent to a previous fusion. Spine 1997;22(14):1574-9.

18. Acosta FL Jr, Ames CP. Cervical disc arthroplasty: general introduction. Neurosurg Clin N Am 2005;16(4):603-7.

19. Pimenta L, McAfee PC, Cappuccino A, et al. Clinical experience with the new artificial cervical PCM (Cervitech) disc. Spine J 2004;4(6 Suppl):315S-21S.

20. Durbhakula MM, Ghiselli G. Cervical total disc replacement, part I: rationale, biomechanics, and implant types. Orthop Clin North Am 2005;36(3):349-54.

21. Mummaneni PV, Burkus JK, Haid RW, Traynelis VC, Zdeblick TA. Clinical and radiographic analysis of cervical disc arthroplasty compared with allograft fusion: a randomized controlled clinical trial. J Neurosurg Spine 2007;6(3):198-209.

22. McAfee PC. The indications for lumbar and cervical disc replacement. Spine J 2004;4(6 Suppl):177S-81S.

23. Sekhon LH. Cervical arthroplasty in the management of spondylotic myelopathy: 18-month results. Neurosurg Focus 2004;17(3):E8.

24. Wang Y, Zhang X, Xiao S, et al. Clinical report of cervical arthroplasty in management of spondylotic myelopathy in Chinese. J Orthop Surg 2006;1:13.

25. Pimenta L, McAfee PC, Cappuccino A, et al. Superiority of multilevel cervical arthroplasty outcomes versus single-level outcomes: 229 consecutive PCM prostheses. Spine 2007;32(12):1337-44.

26. Traynelis VC. The Prestige cervical disc. Neurosurg Clin N Am 2005;16(4):621-8.

27. Chi JH, Ames CP, Tay B. General considerations for cervical arthroplasty with technique for ProDisc-C. Neurosurg Clin N Am 2005;16(4):609-19.

28. Papadopoulos S. The Bryan cervical disc system. Neurosurg Clin N Am 2005;16(4):629-36.
29. Mummaneni PV, Robinson JC, Haid RW Jr. Cervical arthroplasty with the PRESTIGE LP cervical disc. Neurosurgery 2007;60(4 Suppl 2):310-4.

30. Link HD, McAfee PC, Pimenta L. Choosing a cervical disc replacement. Spine J 2004;4(Suppl 6):294S-302S.

31. Sekhon LH, Duggal N, Lynch JJ, et al. Magnetic resonance imaging clarity of the Bryan, Prodisc-C, Prestige LP, and PCM cervical arthroplasty devices. Spine 2007;32(6):673-80.

32. Traynelis VC. The Prestige cervical disc replacement. Spine J 2004;4(6 Suppl):310S-4S.

33. Singh K, An HS. Motion preservation technologies: alternatives to spinal fusion. Am J Orthop 2006;35(9):411-6.

34. Orr RD, Postak PD, Rosca M, et al. The current state of cervical and lumbar spinal disc arthroplasty. J Bone Joint Surg Am 2007;89:70-5.

35. Bryan VE. Jr Cervical motion segment replacement. Eur Spine J 2002;11(Suppl 2):S92-7.

36. Lafuente J, Casey AT, Petzold A, et al. The Bryan cervical disc prosthesis as an alternative to arthrodesis in the treatment of cervical spondylosis: 46 consecutive cases. J Bone Joint Surg Br 2005;87(4):508-12.

37. Anderson PA, Sasso RC, Rouleau JP, et al. The Bryan cervical disc: wear properties and early clinical results. Spine J 2004;4(6 Suppl):303S-9S.

38. Porchet F, Metcalf NH. Clinical outcomes with the Prestige II cervical disc: preliminary results from a prospective randomized clinical trial. Neurosurg Focus 2004;17(3):E6.

39. Wang MY, Leung $\mathrm{CH}$, Casey AT. Cervical arthroplasty with the Bryan disc. Neurosurgery 2005;56(1 Suppl):58-65.

40. Wigfield CC, Gill SS, Nelson RJ, et al. The new Frenchay artificial cervical joint: results from a two-year pilot study. Spine 2002;27(22):2446-52.

41. Hacker RJ. Cervical disc arthroplasty: a controlled randomized prospective study with intermediate follow-up results. J Neurosurg Spine 2005;3(6):424-8.

42. Nabhan A, Ahlhelm F, Shariat K, et al. The ProDisc-C prosthesis: clinical and radiological experience 1 year after surgery. Spine 2007;32(18):1935-41.

43. Sasso RC, Smucker JD, Hacker RJ, et al. Clinical outcomes of BRYAN cervical disc arthroplasty: a prospective, randomized, controlled, multicenter trial with 24-month follow-up. J Spinal Disord Tech 2007;20(7):481-91.

44. Bertagnoli R, Yue JJ, Pfeiffer F, et al. Early results after ProDiscC cervical disc replacement. J Neurosurg Spine 2005;2(4):40310.

45. Goffin J, Van Calenbergh F, van Loon J, et al. Intermediate follow-up after treatment of degenerative disc disease with the Bryan Cervical Disc Prosthesis: single-level and bi-level. Spine 2003;28(24):2673-8.

46. Bertagnoli R, Duggal N, Pickett GE, et al. Cervical total disc replacement, part two: clinical results. Orthop Clin North Am 2005;36(3):355-62.

47. Robertson JT, Metcalf NH. Long-term outcome after implantation of the Prestige I disc in an end-stage indication: 4-year results from a pilot study. Neurosurg Focus 2004;17(3):E10.

48. Pickett GE, Sekhon LH, Sears WR, et al. Complications with cervical arthroplasty. J Neurosurg Spine 2006;4(2):98-105.

49. Pracyk JB, Traynelis VC. Treatment of the painful motion segment: cervical arthroplasty. Spine 2005;30(16 Suppl):S23-32. 